\title{
Correlation-Induced Resonances in Transport through Coupled Quantum Dots
}

\author{
V. Meden ${ }^{1}$ and F. Marquardt ${ }^{2}$ \\ ${ }^{1}$ Institut für Theoretische Physik, Universität Göttingen, D-37077 Göttingen, Germany \\ ${ }^{2}$ Sektion Physik, Universität München, D-80333 München, Germany
}

(Received 26 October 2005; published 11 April 2006)

\begin{abstract}
We investigate the effect of local electron correlations on transport through parallel quantum dots. The linear conductance as a function of gate voltage is strongly affected by the interplay of the interaction $U$ and quantum interference. We find a pair of novel correlation-induced resonances separated by an energy scale that depends exponentially on $U$. The effect is robust against a small detuning of the dot energy levels and occurs for arbitrary generic tunnel couplings. It should be observable in experiments on the basis of presently existing double-dot setups.
\end{abstract}

DOI: 10.1103/PhysRevLett.96.146801

The theoretical and experimental research on electronic transport through ultrasmall quantum dots has become a very active field. Various fundamental physical phenomena, such as quantum interference [1], Coulomb blockade [2], and the Kondo effect [3,4], strongly affect the transport properties. Currently, the focus is shifting towards few-electron double-dot structures that are studied as artificial molecules, interferometers, and for charge- and spin-based quantum computing [5-13]. Investigations into the role of correlations in systems of two or more coupled quantum dots are still at the beginning and much remains to be explored. In parallel quantum dots connected to common leads, new physics is to be expected due to the interplay of correlations and quantum interference.

In the present Letter, we investigate this problem for a specific model of two parallel quantum dots coupled by an electron interaction $U$ (see Fig. 1). The effect of external electrostatic potentials on two-path interference was studied earlier (magnetoelectric Aharonov-Bohm effect) [14]. We here investigate the role of interaction-induced potentials. This is especially relevant for molecular transport, as the interaction-induced potentials will be far larger than the external potentials which are difficult to apply to such small structures. We study how the linear conductance $G$ as a function of gate voltage $V_{g}$ changes with increasing $U$. Considering the entire parameter space, we find a very rich generic behavior and predict the appearance of novel correlation-induced resonances (CIRs) if $U$ is larger than a critical interaction $U_{c}$. The effect is robust: It appears for almost arbitrary combinations of the four tunnel couplings and also remains visible for a small detuning of the dot level energies. The separation of the resonances in gate voltage defines an energy scale that depends exponentially on $U$ and on a combination of the tunnel couplings. It is argued that this new correlation effect is unrelated to Kondo physics. We employ a powerful new method, the functional renormalization group (FRG), to efficiently obtain both numerical and analytical results for this manybody problem, and we have confirmed all the essential features using the numerical renormalization group (NRG). Double-dot geometries that could form the basis
PACS numbers: 73.63.Kv, 73.23.Hk

to verify our predictions have been experimentally realized in Refs. [7-10]. Our model is equally of relevance for transport through two nearly degenerate levels of a single dot, a subject that has attracted much attention recently in attempts to understand the puzzling behavior of the transmission phase [1].

We study a system of two quantum dots $j=1,2$ each having a single level $\varepsilon_{j}$ as sketched in Fig. 1. The dots are coupled by a Coulomb interaction $U \geq 0$ and are connected to two common leads $l=L, R$ via tunnel barriers $t_{j}^{l}$. The dot Hamiltonian is $H_{\mathrm{dot}}=\sum_{j} \varepsilon_{j} d_{j}^{\dagger} d_{j}+U\left(n_{1}-\right.$ $1 / 2)\left(n_{2}-1 / 2\right)$ and the dot-lead coupling is given by $H_{T}=-\sum_{j, l}\left(t_{j}^{l} c_{0, l}^{\dagger} d_{j}+\right.$ H.c. $)$, where $c_{0, l}^{\dagger}$ denotes the creation operator at the end of the semi-infinite lead $l$. The leads are modeled by $H_{l}=-t \sum_{m=0}^{\infty}\left(c_{m, l}^{\dagger} c_{m+1, l}+\right.$ H.c. $)$. The energy scale of the dot level broadening is given by $\Gamma_{j}^{l}=\pi\left|t_{j}^{l}\right|^{2} \rho_{l}$, where $\rho_{l}$ denotes the local density of states at the end of lead $l$. As usual, we later take $\rho_{l}$ to be energy independent. The ring structure is pierced by a magnetic flux $\phi$ that we take into account by multiplying $t_{2}^{R}$ by a phase factor $e^{i \phi}$. For symmetry reasons, one only has to consider $0 \leq \phi \leq \pi$. The levels are shifted by a common gate voltage $V_{g}$ such that $\varepsilon_{1}=V_{g}+\delta$ and $\varepsilon_{2}=V_{g}$, where $\delta$ denotes a detuning. For $\delta=0$ due to the shift of $n_{j}$ in $H_{\text {dot }}, V_{g}=0$ corresponds to half-filled dots. We neglect the spin of the electron and, thus, suppress the spin Kondo effect. Experimentally, the contribution of spin physics may be excluded by applying a sufficiently strong magnetic field [10]. We focus on temperature $T=0$. The spectral properties [15] and level occupancies [16,17] of this model were investigated earlier.

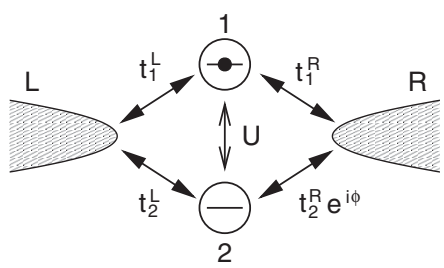

FIG. 1. System of coupled quantum dots with common leads. 
To compute $G$ and the level occupancies $\left\langle n_{j}\right\rangle$, we use mainly a recently developed FRG scheme [18]. The starting point is an exact hierarchy of differential flow equations for the self-energy $\Sigma^{\Lambda}$ and higher order vertex functions, where $\Lambda \in(\infty, 0]$ denotes an infrared energy cutoff which is the flow parameter. We truncate the hierarchy by neglecting the flow of the two-particle vertex only considering $\Sigma^{\Lambda}$, which is then energy independent. This approximation and variants of it were successfully used to study quasi-one-dimensional wires of correlated electrons (Tomonaga-Luttinger liquids) [19] and locally correlated systems [20].

For the double dot, $\Sigma^{\Lambda}$ is a $2 \times 2$ matrix in the dot label $j$. The diagonal parts are real and $V_{j}^{\Lambda}=\Sigma_{j, j}^{\Lambda}+V_{g}$ can be considered as effective dot level positions. The (for arbitrary $\phi$ ) complex off-diagonal contribution $t_{d}^{\Lambda}=-\Sigma_{1,2}^{\Lambda}$ is a hopping between the two dot states generated by the interaction. The flow equations are

$$
\begin{aligned}
\partial_{\Lambda} V_{j}^{\Lambda} & =-\frac{U}{2 \pi} \sum_{\omega= \pm \Lambda} G_{\bar{j}, \bar{j}}^{\Lambda}(i \omega), \\
\partial_{\Lambda} t_{d}^{\Lambda} & =-\frac{U}{2 \pi} \sum_{\omega= \pm \Lambda} G_{1,2}^{\Lambda}(i \omega),
\end{aligned}
$$

with $\bar{j}$ being the complement of $j$. The Green function is $G^{\Lambda}(i \omega)=\left[i \omega-h^{\Lambda}(i \omega)\right]^{-1}$, with

$$
h^{\Lambda}(i \omega)=\left(\begin{array}{cc}
V_{1}^{\Lambda}-i \Gamma_{1} \operatorname{sgn}(\omega) & -t_{d}^{\Lambda}-i \gamma \operatorname{sgn}(\omega) \\
-\left(t_{d}^{\Lambda}\right)^{*}-i \gamma^{*} \operatorname{sgn}(\omega) & V_{2}^{\Lambda}-i \Gamma_{2} \operatorname{sgn}(\omega)
\end{array}\right)
$$

and $\Gamma_{j}=\sum_{l} \Gamma_{j}^{l}, \quad \gamma=\sqrt{\Gamma_{1}^{L} \Gamma_{2}^{L}}+e^{i \phi} \sqrt{\Gamma_{1}^{R} \Gamma_{2}^{R}}$. The initial conditions are $V_{1}^{\Lambda=\infty}=V_{g}+\delta, V_{2}^{\Lambda=\infty}=V_{g}$, and $t_{d}^{\Lambda=\infty}=$ 0 . To obtain an approximation for $\Sigma$ and thus $G$, one has to solve the system (1) of four real coupled differential equations. This can easily be done numerically and for a specific class of parameters also analytically. The occupancies $\left\langle n_{j}\right\rangle$ can directly be calculated from $\mathcal{G}$. For the present problem, the same holds for $G$ as current vertex corrections vanish. One can easily derive a lengthy expression for $G$ in terms of the parameters $\Gamma_{j}^{l}, \phi$ and the renormalized level positions $V_{j}=V_{j}^{\Lambda=0}$ and hopping $t_{d}=t_{d}^{\Lambda=0}$ not presented here. $V_{j}$ and $t_{d}$ depend on $\Gamma_{j}^{l}, \phi$ as well as on $V_{g}, \delta$, and $U$.

We use the NRG as a nonperturbative method to confirm the essential validity of the physics discovered within the FRG. To compute the conductance for arbitrary dot-lead couplings, we apply the Kubo formula

$$
\operatorname{Re} G(\omega)=\frac{\pi}{\omega} \sum_{f}\left|I_{0, f}\right|^{2} \delta\left(E_{f}-E_{0}-\omega\right), \quad \omega>0 .
$$

The current operator $I=e\left(\dot{N}_{R}-\dot{N}_{L}\right) / 2$ (with $N_{l}=$ $\sum_{m=0}^{\infty} c_{m, l}^{\dagger} c_{m, l}$ and $\dot{N}_{l}=i\left[H, N_{l}\right]$ ) is expressed in terms of lead and dot operators. Its matrix elements $I_{0, f}$ (with 0 indicating the ground state and $f$ the excited states; $E_{0}$ and $E_{f}$ are the respective energies) are evaluated in the NRG basis and the $\omega \rightarrow 0$ limit then provides the dc conductance $G$ [21].
We first consider the case of degenerate levels with $\delta=$ 0 . The behavior of $G\left(V_{g}, U\right)$ can be cast in four classes that can already be identified at $U=0$. (i) If two or more of the $\Gamma_{j}^{l}$ are 0 such that no closed path between the left and right leads exists $G\left(V_{g}, U\right) \equiv 0$. (ii) $\Gamma_{1}^{L} \Gamma_{1}^{R}=\Gamma_{2}^{L} \Gamma_{2}^{R}$ and $\phi=\pi$ : In this case, one can introduce new fermionic dot states such that one couples only to the left lead and the other only to the right lead, implying $G\left(V_{g}, U\right) \equiv 0$. We note that in this case (and only in this case) a conserved pseudospin variable (left/right) exists [15]. (iii) A nonvanishing (but nongeneric) conductance is found for $\Gamma_{1}^{L} \Gamma_{2}^{R}=\Gamma_{1}^{R} \Gamma_{2}^{L} \neq 0$ and $\phi=0$. In this case, $G\left(V_{g}, U=0\right)$ is given by a Lorentzian centered around 0 . The $U>0$ dependence of $G\left(V_{g}, U\right)$ can most easily be studied in the exactly solvable case of equal $\Gamma_{j}^{l}$ [15]. It is characterized by two Coulomb blockade peaks located at $\approx \pm U / 2$. (iv) For all other $\Gamma_{j}^{l}$, $\phi$, that is, for generic parameters on which we focus here, the peak in $G\left(V_{g}, U=0\right)$ at $V_{g}=0$ [as in case (iii)] is replaced by a dip with $G\left(V_{g}, U=0\right)=0$. For equal $\Gamma_{j}^{l}$, $\phi=0, \delta>0$, and $U=0$, the appearance of such dips was discussed earlier and explained as a destructive interference between path traversing dots 1 and 2, respectively [22]. In the limit of a strong asymmetry of the transmission probability via dots 1 and 2, e.g., for $\Gamma_{1}^{l} \ll \Gamma_{2}^{l}$, the dip can be viewed as a Fano antiresonance resulting from the interference of a resonant path and a path with energy independent transmission.

Figure 2 shows the generic evolution of $G\left(V_{g}\right)$ for increasing $U$ at $\delta=0$. Because of particle-hole symmetry, $G$ is symmetric around $V_{g}=0$. Energies are given in units of $\Gamma=\sum_{j} \Gamma_{j}$. Increasing $U$, the height of the two peaks resulting from the dip at $V_{g}=0$ increases and the maximum flattens. At a critical $U=U_{c}\left(\left\{\Gamma_{j}^{l}\right\}, \phi\right)$, each of the

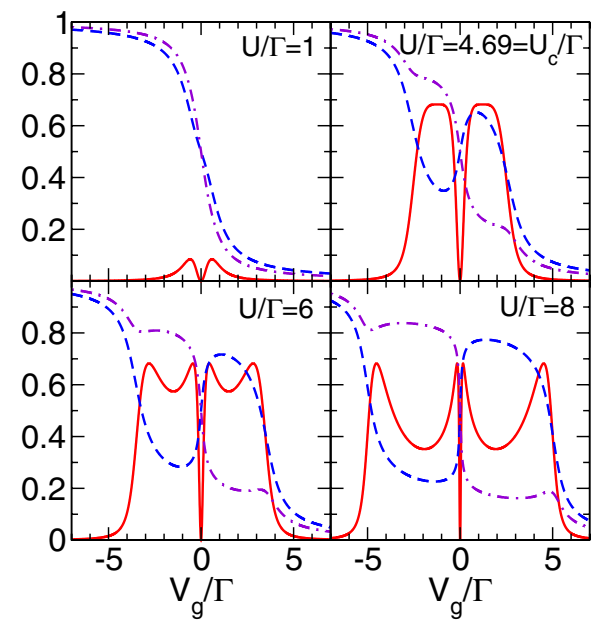

FIG. 2 (color online). Generic results for $G\left(V_{g}\right) /\left(e^{2} / h\right)$ (solid lines), $\left\langle n_{1}\right\rangle$ (dashed lines), and $\left\langle n_{2}\right\rangle$ (dashed-dotted lines) at different $U$ obtained from the FRG with $\Gamma_{1}^{L}=0.27 \Gamma, \Gamma_{1}^{R}=$ $0.33 \Gamma, \Gamma_{2}^{L}=0.16 \Gamma, \Gamma_{2}^{R}=0.24 \Gamma, \phi=\pi$, and $\delta=0$. The two novel correlation-induced resonances are visible in the lower panels (large $U$ ), near $V_{g}=0$. 
peaks splits into two. Further increasing $U$, the two outermost peaks move towards larger $\left|V_{g}\right|$ and become the Coulomb blockade peaks located at $V_{g} \approx \pm U / 2$. The other two peaks at $\pm V_{\mathrm{CIR}}$ are the novel CIRs, where $V_{\mathrm{CIR}}>$ 0 decreases with increasing $U$. Associated with $G\left(V_{g}=\right.$ $0)=0$ at $U=0$ is a jump of the transmission phase by $\pi$. As the phase evolves continuously with $U$ and particlehole symmetry holds for any $U$, the $\pi$-phase jump and thus $G\left(V_{g}=0\right)=0$ must remain for all $U$. For $U>U_{c}$, the height of all four peaks is equal to $h_{\max }\left(\left\{\Gamma_{j}^{l}\right\}, \phi\right) \leq e^{2} / h$ and does not change with $U$.

For equal $\Gamma_{j}^{l}, \phi=\pi$, and $\delta=0$ [case (ii) above], the conserved pseudospin leads to orbital Kondo physics [15]. Remnants of this effect were found in the vicinity of this parameter point but die out quickly away from it. As our correlation effect appears generically, in particular, for parameters far away from the Kondo point, it is apparently unrelated to Kondo physics.

In addition to $G\left(V_{g}\right)$ in Fig. 2, we present the dot occupancies. For small $U$, the $V_{g}$ dependence of the occupancies of dots 1 and 2 is monotonic. In the opposite limit of large $U,\left\langle n_{j}\right\rangle$ depends nonmonotonically on $V_{g}[16,17]$. Starting at negative $V_{g}$, the level that is coupled more strongly (here $j=1$ ) is depopulated at the first Coulomb blockade peak, while the occupancy of the other level stays close to 1 . Close to $V_{g}=0$, we find an inversion of the population followed by another depopulation of the more strongly coupled dot across the second Coulomb blockade peak at $V_{g} \approx U / 2$. Note that the nonmonotonic behavior of the $\left\langle n_{j}\right\rangle$ sets in for interactions smaller than $U_{c}$ and is, thus, not directly related to the appearance of the CIRs. This is consistent with the observation that, in contrast to the CIRs (see below), the nonmonotonicity of $\left\langle n_{j}\right\rangle$ can already be observed within a self-consistent Hartree approximation [16].

The left part of Fig. 3 shows a comparison of FRG and NRG data for $G$ at $V_{g}>0$ and for different $U$. To clearly resolve the CIRs, we use a logarithmic scale. The NRG data show all the features discussed in connection with Fig. 2. In particular, for increasing $U>U_{c}, V_{\mathrm{CIR}}$ becomes

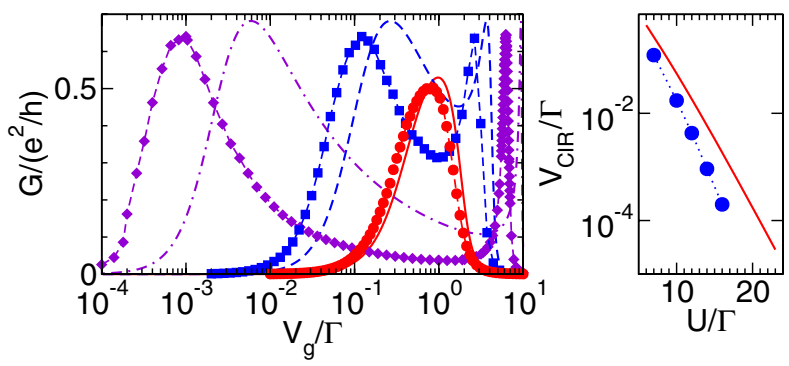

FIG. 3 (color online). Comparison of FRG (lines) and NRG (symbols) results for the same parameters as in Fig. 2. Left: $G\left(V_{g}\right)$ for different $U(U=3.5 \Gamma$ : solid line and circles; $U=7 \Gamma$ : dashed line and squares; $U=14 \Gamma$ : dashed-dotted line and diamonds). Right: The resonance position $V_{\mathrm{CIR}}$. small quickly (right part of Fig. 3). For $U$ sufficiently larger than $U_{c}, V_{\mathrm{CIR}} / \Gamma \propto \exp \left[-C\left(\left\{\Gamma_{j}^{l}\right\}, \phi\right) U / \Gamma\right]$, with $C>0$. By construction, the FRG works well up to intermediate $U$. At larger $U$ and for all $\Gamma_{j}^{l}$, $\phi$, we tested the FRG overestimates the positions of the Coulomb blockade peaks and the CIRs. As will be shown in an upcoming publication, this can systematically be improved using a more elaborate FRG truncation scheme. For a specific class of $\Gamma_{j}^{l}$, $\phi$, we next analytically confirm the exponential dependence of $V_{\mathrm{CIR}}$ on $U$ and derive an explicit expression for $C$ using the FRG. In the most general case, the dependence of $C$ (and $U_{c}$ ) on $\Gamma_{j}^{l}$ is complex and requires further investigation. Roughly speaking, $C$ increases $\left(U_{c}\right.$ decreases) with increasing asymmetry of the $\Gamma_{j}^{l}$ [see also Eq. (4) below]. For fixed $\Gamma_{j}^{l}$ and increasing $0 \leq \phi \leq \pi, C$ decreases while $U_{c}$ increases.

We now consider $\Gamma_{1}^{L}=\Gamma_{1}^{R}, \Gamma_{2}^{L}=\Gamma_{2}^{R}$, but $\Gamma_{1}^{L} \neq \Gamma_{2}^{L}$, and $\phi=\pi$. In this case, $\gamma=0$ in Eq. (2) and the off-diagonal elements of the Green function $\mathcal{G}^{\Lambda}$ are proportional to $t_{d}^{\Lambda}$. Initially (at $\Lambda=\infty$ ), $t_{d}^{\Lambda}$ vanishes and it will thus remain zero during the FRG flow, which leads to a simplification of the flow equation (1). For small $V_{j}^{\Lambda}$, that is, small $V_{g}$, these equations can be solved analytically, and in the limit $U \gg\left|\Gamma_{1}^{L}-\Gamma_{2}^{L}\right|$ we obtain

$$
V_{\mathrm{CIR}} / \Gamma \propto \exp \left[-\frac{U}{2 \pi} \frac{\ln \left(\Gamma_{1}^{L} / \Gamma_{2}^{L}\right)}{\Gamma_{1}^{L}-\Gamma_{2}^{L}}\right]
$$

In Fig. 4, we show the renormalized level positions $V_{j}$ as a function of $V_{g}$ for fixed $U>U_{c}$. For $\left|V_{g}\right| \gg U / 2, V_{1}$ and $V_{2}$ become equal and are given by $V_{g}-\operatorname{sgn}\left(V_{g}\right) U / 2$. For $-U / 2 \lesssim V_{g}<0$, the position of the level that is coupled more weakly (here $j=2$ ) is smaller than the chemical potential $\mu=0$, while the other level has energy larger than $\mu$. For $0<V_{g} \lesssim U / 2$, the role of the two levels is interchanged. This explains the observed $V_{g}$ dependence of the $\left\langle n_{j}\right\rangle$ discussed in connection with Fig. 2. Peaks in $G$ are found at $V_{1} V_{2}=-4 \Gamma_{1}^{L} \Gamma_{2}^{L}$. In particular, the crossings of

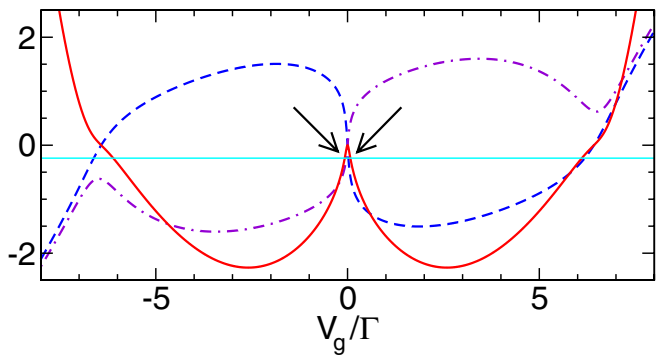

FIG. 4 (color online). Renormalized dot level positions $V_{1} / \Gamma$ (dashed line) and $V_{2} / \Gamma$ (dashed-dotted line). The thick solid line is the product $V_{1} V_{2} / \Gamma^{2}$. The thin solid line lies at $-4 \Gamma_{1}^{L} \Gamma_{2}^{L} / \Gamma^{2}$, and its intersections with the thick solid line determine the peak positions of $G$. The two crossings indicated by the arrows are at $V_{g}= \pm V_{\mathrm{CIR}}$. The parameters are $U=10 \Gamma, \Gamma_{1}^{L}=\Gamma_{1}^{R}=0.3 \Gamma$, $\Gamma_{2}^{L}=\Gamma_{2}^{R}=0.2 \Gamma, \phi=\pi$, and $\delta=0\left(U_{c} / \Gamma \approx 5.05\right.$ from FRG). 


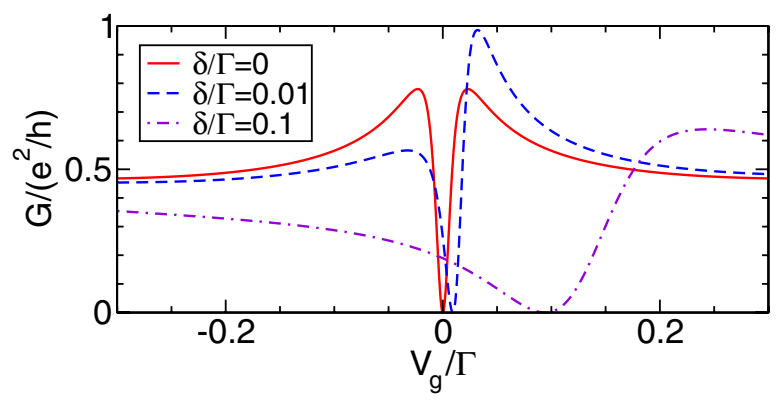

FIG. 5 (color online). Dependence of $G\left(V_{g}\right)$ on the level splitting $\delta$. The parameters are $U=3 \Gamma, \Gamma_{1}^{L}=0.7 \Gamma, \Gamma_{1}^{R}=$ $0.2 \Gamma, \Gamma_{2}^{L}=0.02 \Gamma, \Gamma_{2}^{R}=0.08 \Gamma$, and $\phi=0\left(U_{c} / \Gamma \approx 0.842\right.$ from FRG).

$V_{1} V_{2}$ (thick solid line) and $-4 \Gamma_{1}^{L} \Gamma_{2}^{L}$ (thin solid line) at $\pm V_{\mathrm{CIR}}$ occur because $V_{1}$ and $V_{2}$ continuously go through 0 at $V_{g}=0$, one coming from above, the other from below. This is not the case if the self-consistent Hartree-Fock approximation is used to compute the effective level positions. Within this approach, $V_{1} V_{2}$ discontinuously jumps at $V_{g}=0$ leading to only two crossings at $V_{g} \approx \pm U / 2$ associated with the Coulomb blockade peaks. This shows that, similar to the Kondo effect, more sophisticated methods than the Hartree-Fock approximation are required to describe local correlation effects.

We now investigate the stability of the CIRs in the presence of an initial level splitting $\delta>0$. In Fig. 5, we show FRG data for $G\left(V_{g}\right)$ with different $\delta$ at small $\left|V_{g}\right|$. For $\delta>\Gamma$ and all parameter sets we studied, $G$ shows only the two Coulomb blockade peaks at $V_{g} \approx-U / 2-\delta$ and $V_{g} \approx U / 2$. The way this large $\delta$ limit is reached depends on the specific choice of parameters, but, common to all cases, remnants of the CIRs were clearly observable for $\delta \ll \Gamma$, as exemplified in Fig. 5. We found a similar $\delta$ dependence using NRG. A direct hopping between the two dots induces a level splitting and has thus an effect similar to that of $\delta>0$.

To summarize, we found that the transport properties of a double-dot interferometer are strongly affected by electron correlations. We discovered a novel pair of correlation-induced resonances that should be measurable in double dots of appropriate geometry [7-10] in the presence of strong Zeeman splitting. Varying the tunnel barriers (and thus $U / \Gamma$ ), it should be possible to study the entire scenario discussed above. Apparently, this correlation effect is unrelated to both spin [3] and orbital [15] Kondo physics. Rather, it follows from the interplay of local correlations and quantum interference. It is thus likely that similar effects will appear in transport through more complex systems as, e.g., ringlike molecules studied in the context of molecular electronics. Besides revealing interesting new physics, we showed that the FRG method is a very promising tool to investigate problems with local Coulomb correlations. In comparison to NRG, FRG is far superior in terms of the numerical effort required, e.g., enabling efficient analysis of parameter dependencies. Furthermore, the FRG can easily be extended to more complex systems with local electron correlations.

We thank J.v. Delft, T. Enss, W. Hofstetter, Th. Pruschke, H. Schoeller, K. Schönhammer, A. Sedeki, and A. Weichselbaum for valuable discussions and, in particular, M. Sindel for supplying us with his NRG code. V.M. is grateful to the Deutsche Forschungsgemeinschaft (SFB 602) for support.

[1] R. Schuster et al., Nature (London) 385, 417 (1997); M. Avinun-Khalish et al., Nature (London) 436, 529 (2005), and references therein.

[2] Mesoscopic Electron Transport, edited by L. L. Sohn, L. P. Kouwenhoven, and G. Schön (Kluwer, Dodrecht, 1997).

[3] L. Glazman and M. Raikh, JETP Lett. 47, 452 (1988); T. Ng and P. Lee, Phys. Rev. Lett. 61, 1768 (1988).

[4] D. Goldhaber-Gordon et al., Nature (London) 391, 156 (1998); W. van der Wiel et al., Science 289, 2105 (2000).

[5] D. Loss and D. P. DiVincenzo, Phys. Rev. A 57, 120 (1998); D. Loss and E. V. Sukhorukov, Phys. Rev. Lett. 84, 1035 (2000).

[6] F. Marquardt and C. Bruder, Phys. Rev. B 68, 195305 (2003).

[7] A. W. Holleitner et al., Phys. Rev. Lett. 87, 256802 (2001); Science 297, 70 (2002).

[8] J.C. Chen, A. M. Chang, and M. R. Melloch, Phys. Rev. Lett. 92, 176801 (2004).

[9] M. Sigrist et al., Phys. Rev. Lett. 93, 066802 (2004).

[10] A. W. Holleitner et al., Phys. Rev. B 70, 075204 (2004).

[11] J. R. Petta et al., Phys. Rev. Lett. 93, 186802 (2004).

[12] F. H. L. Koppens et al., Science 309, 1346 (2005).

[13] J. R. Petta et al., Science 309, 2180 (2005).

[14] Yu. V. Nazarov, Phys. Rev. B 47, 2768 (1993); A. van Oudenaarden, M. H. Devoret, Yu. V. Nazarov, and J.E. Mooij, Nature (London) 391, 768 (1998); W. G. van der Wiel et al., Phys. Rev. B 67, 033307 (2003).

[15] D. Boese, W. Hofstetter, and H. Schoeller, Phys. Rev. B 64, 125309 (2001).

[16] M. Sindel, A. Silva, Y. Oreg, and J. von Delft, Phys. Rev. B 72, 125316 (2005).

[17] J. König and Y. Gefen, Phys. Rev. B 71, 201308(R) (2005).

[18] V. Meden, W. Metzner, U. Schollwöck, and K. Schönhammer, J. Low Temp. Phys. 126, 1147 (2002).

[19] V. Meden et al., Europhys. Lett. 64, 769 (2003); T. Enss et al., Phys. Rev. B 71, 155401 (2005); X. BarnabéThériault, A. Sedeki, V. Meden, and K. Schönhammer, Phys. Rev. Lett. 94, 136405 (2005).

[20] R. Hedden, V. Meden, Th. Pruschke, and K. Schönhammer, J. Phys. Condens. Matter 16, 5279 (2004); S. Andergassen, T. Enss, and V. Meden, cond-mat/0509576.

[21] M. Sindel, W. Hofstetter, J. von Delft, and M. Kindermann, Phys. Rev. Lett. 94, 196602 (2005).

[22] A. Silva, Y. Oreg, and Y. Gefen, Phys. Rev. B 66, 195316 (2002). 